\title{
Toward the development of standardized procedures for structural health monitoring
}

\begin{tabular}{|c|c|}
\hline Journal: & IABSE Guimaraes 2019 \\
\hline Manuscript ID & GUI-0308-2019.R1 \\
\hline Theme: & Lifecycle Quality Control of New and Existing Infrastructures \\
\hline $\begin{array}{l}\text { Date Submitted by the } \\
\text { Author: }\end{array}$ & $\mathrm{n} / \mathrm{a}$ \\
\hline Complete List of Authors: & $\begin{array}{l}\text { Limongelli, Maria Pina; Politrcnico di Milano, Architecture, Built } \\
\text { Environment, Construction Engineering }\end{array}$ \\
\hline Keywords: & $\begin{array}{l}\text { Codes and Standards }<\text { Other Aspects, Concrete }<\text { Material and } \\
\text { Equipment, Dynamic effects/vibrations }<\text { Other Aspects }\end{array}$ \\
\hline
\end{tabular}

\section{SCHOLARONE \\ Manuscripts}




\title{
Toward the development of standardized procedures for structural health monitoring
}

\author{
Maria Pina Limongelli \\ Politecnico di Milano, Milano, Italy \\ Contacting author: mariagiuseppina.limongelli@polimi.it
}

\section{Abstract}

Monitoring of structural health conditions is performed using different methods that range from periodic surveys including nondestructive testing at selected locations, to permanent monitoring using network of sensors continuously recording the structural response. These procedures aim at providing detection of possible faults or deterioration processes in order to optimally manage civil structures and infrastructures over the lifecycle. To date several guidelines have been published by different countries all over the world but protocols to apply SHM are generally not defined nor enforced. This is likely to be of the reasons that stand behind the limited diffusion and implementation of SHM for routine operations of condition assessment. In this paper building the principal aspects of the SHM process are presented and the need of the development of protocols for the different phases of the SHM process, from design to practical implementation and use are outlined.

Keywords: Structural Health Monitoring, condition monitoring, standardization, indicators, data.

\section{Introduction}

Structural Health Monitoring (SHM) consists in the use of measurements collected on the structure to identify parameters representatives of the structural condition or of the external actions. The evolution in time of these parameters allows to identify the possible onset of damage or degradation and to characterize their uncertainty.

SHM is performed using different methods that range from periodic surveys, including visual inspections and nondestructive tests at selected locations, to permanent monitoring using network of sensors that continuously record the structural response. Visual inspections are the traditional method of survey, they are usually performed periodically to check degradation phenomena or after extreme events to detect possible damages. The outcome of this type of inspection can be sensitive to the experience of the operator and not able to detect hidden faults. Nondestructive tests are carried out during more refined surveys when the vicinity of damage is already known and provide a deeper knowledge about local faults. Permanent monitoring systems are usually based on the measurement of the structural response to ambient vibrations and have the advantage, with respect to the previous inspection methods, of providing global information about the structural conditions. This enables the detection of hidden damages even if their location is not previously known or is not accessible.

Information from SHM systems play a significant role in supporting condition assessment and proactive maintenance aimed to a) prevent failures and b) detect the onset damages before they impact the performance of the system.

Notwithstanding several successful applications and important efforts on behalf of researchers, 
SHM is not yet extensively and routinely used for condition assessment of civil structures.

Several reasons stand behind this. One is the insufficient knowledge and understanding of the process on behalf of the stakeholders (from owners to sensors producers) [1]. Another is the fact that only recently efforts have been invested in procedures to quantify the value of an SHM system before its implementation [2]. The difficulty to estimate the return on investment associated with the SHM the implementation of a monitoring system has often caused reluctance of the owners in investing in these technologies. Another reason that has hampered in the past a diffused implementation of SHM systems might be the lack of standardized protocols for their design and implementation.

In the last years there have been several efforts all over the world to outline guidelines and recommendations for SHM for Civil Structures. The first SHM guidelines, where published in Canada in 2001 [4] followed in 2006 by the SAMCO recommendations in Europe [5] Recently in China the first, at the author knowledge, compulsory technical code was enforced [6].

In 2014 the Italian Institute for Standard (UNI) prepared a Technical Report with the guidelines for the design, Installation, maintenance and operation of SHM for Civil Engineering Structures [1]. Several protocols for the collection of data, visual inspection, sampling and testing of materials, nondestructive evaluation, and data storage and management was published in 2016 by the Federal Highway Administration of the US Department of Transportation [7], [8].

The Action Group AG9 on 'Testing and Structural Health Monitoring' the contributions on the last development on these topics to include in the Model Code 2020.

The standardization of SHM procedures shall tackle several aspects involved in the SHM process:

a) provide guidelines on what type of functionalities can be investigated with specific tests;

b) provide guidelines on how to obtain indicators relevant for the assessment of the structural condition with respect to a given functionality; c) provide performance goals (thresholdsor minimum/maximum requirements) for specific indicators;

d) suggest tools for data collection (measurement, transmission, processing) able to ensure consistency of the data and the possibility to compare data obtained using different techniques;

e) specify minimum requirements for the different types of sensors (from their characteristics to the requirements to follow for their installation);

f) describe potential shortcomings of specific techniques for data collection;

g) specify minimum requirements for the procedures for data transmission and processing.

A consistent definition of the previous points may allow to clearly define the capabilities of different procedures for SHM therefore enabling the stakeholders to perform a correct choice among different systems depending on their needs.

In the following sections, in order to facilitate the description and the needs in terms of standardization, connected with the different aspects involved in the SHM process, a possible step-wise procedure is outlined for the design of an SHM program.

\section{SHM as a decision support tool}

A key purpose of SHM is the need to inform decision making related to an efficient (usually from a cost/benefit balance perspective) management of the structural maintenance throughout its entire life cycle. This requires:

- to manage risk, that is to maximize structure reliability/availability (reduction of direct and indirect costs related to failure/unavailability);

- to reduce the cost of maintenance over life-cycle for example moving from preventive to condition-based maintenance programs (savings in maintenance hours, parts, etc.).

In other terms, one of the main drivers for performing SHM is to minimize risks of functionality failure in the most cost-effective manner over the life cycle. 
Uncertainties related to the structural conditions affect the estimation of both the probability of functionality failure and of the costs related to different consequences. Knowledge about the presence of defects and their severity may be increased by collecting information on the asset through SHM activities: surveys, inspections, tests and monitoring.

This information must be then used to establish if the asset, in its current condition, is able to provide the functionalities required or instead some repair actions should be performed.

To decide whether a procedure for condition monitoring is suitable for a specific case, the stakeholders must understand the characteristics of the procedure e.g. the performance in terms of damage identification, the ease of application and installation of the instruments, the frequency of tests needed.

Therefore, the first step in the definition of a procedure for condition assessment must be the specification of the objectives ad expectations of stakeholders.

Once these aspects are defined, the decision makers can evaluate and compare different condition monitoring procedures and make an educated choice of those most suited for their needs. This can be done based on the experience and knowledge of the decision maker or employing procedures to quantify the value of SHM [2].

If information about loss of stiffness are needed, vibration-based monitoring could be the most effective choice. If tension losses or damage due to corrosion in the prestressing cables of a bridge are the target, vibration based method cannot give direct information about this phenomenon and Non Destructive Testing (NDT) is preferable.

Another variable to consider is the level of insight the stakeholders need. For example, if the only information required is about the existence of damage, the employment of a procedure for damage detection can provide the required information, if the location or the severity of damage are sought, more demanding damage identification procedures, and more sophisticated monitoring tools must be employed.

\section{Design of an SHM program: principal aspects}

The very first step in the definition of an SHM program should be the development of a list of needs (e.g. management of maintenance) and expected information (e.g. development of degrading phenomena) that drive the implementation of an SHM system. Based on this list, the information needed to answer the expectations of the stakeholders can be defined and the data needed to provide this information selected.

Data measured by the sensors are usually translated into a list of indicators used check the structural performance with respect to pre-defined goals. Further steps relate to the design of the monitoring system - based on the characteristics defined at the previous steps - and to the definition of the specific procedures to collect, process and store the data. In the following sections the design of an SHM program will be described with reference to 5 steps:

Step 1. Specify the objectives of and expectations of stakeholders

Step 2. Specify the information the SHM system is expected to require and the data that need to be collected to provide these information

Step 3. Design the monitoring system/program (select monitoring techniques and plan monitoring program to provide the required data minimizing costs)

Step 4. Collect data

Step 5. Process data (assess structural condition) and store information

\subsection{Step 1. Specify the objectives and expectations of stakeholders}

\section{What do stakeholders want to achieve through condition monitoring?}

As mentioned before, when defining an SHM program, the first question to answer relates to needs and expectations of the stakeholders that are the targets driving the condition monitoring. They could be related to the need to ensure the safety of the individual structure or to ensure appropriate functionality minimizing the risk of (safety or serviceability) failure in a cost-effective 
manner. Specific drives for SHM could be the following:

- to assess if the functionality of the structure complies with (regulatory or other) requirements corresponding to specific performance goals (for example safety or availability);

- to prioritize maintenance and rehabilitation interventions according some pre-defined criterion (cost efficiency or importance of the structure)

- to support the planning of emergency and recovery interventions with the aim of reducing losses related to downtime and/or to repeated events;

- to cost-effectively manage maintenance by shifting from a predictive-based to a conditionbased approach.

- To extend the maintenance cycles so reducing downtime related to interventions.

Once the main target is defined, the information needed to reach it can be more easily selected and defined.

\subsection{Step 2. Specify the Information required and the data to collect}

\section{What information are needed from the SHM system?}

The information needed from SHM must support decisions related to the targets specified at step1. This requires the assessment of the structural condition and is usually performed through the use of Indicators able to describe the structural 'fitness for purpose' with respect to the different functionalities of the structure involved in the targets defined at step 1.

For example, if the aim of monitoring is to assess the safety of the structure, the risk of failure can be assumed as indicator whereas indicators related to the structural flexibility could be more useful when the behavior at serviceability limit states is assessed.

Several damage/degradation phenomena may affect and modify the structural functionality requiring decisions about possible rehabilitation and maintenance interventions. In order to describe each of them, several indicators can be computed from data collected through tests or inspections. Examples of Indicators able to provide useful information about losses of stiffness and that can be retrieved from dynamic tests are the modal parameters, (frequency, mode shape, damping ratio) or functions computed form them (modal curvature, modal strain energy, flexibility,...). Other indicators useful to detect stiffness losses can the sag or the displacement profile obtained from static tests. Degradation related to corrosion may be described by local indicators like carbonation depth, chloride content or corrosion rate that can be computed from data collected through NDT.

The indicators must be measurable and for each of them a metric must be defined to assess the structural state against predefined goals that are often defined through threshold values or extreme (minimum or maximum) requirements.

Once the list of the indicators needed to reach the targets at step 1 is compiled, the data needed to compute these indicators must be identified. They can be legacy data (for example archive data related to the original design of the structure or to previous test campaign) or observations collected on the structure by inspections or measurements.

Data must describe the different aspects affecting the structural performance:

- structural details (geometry, mechanical parameters of structural materials and soil);

- existing damage/degradation processes (carbonation depth, pit depths, area reduction, stiffness losses, scour etc.)

- actions on the structures;

- environmental conditions (temperature, moisture content, etc.).

Their temporal and spatial distribution must be defined based on the accuracy requested in the description of the structural performance and accounting for budget constraints. As previously mentioned, the choice between different strategies of data collection can be effectively supported by a Value of Information analyses.

For example, if the requirement of the stakeholders is to detect possible increases of flexibility or a change in the boundary conditions of the structure, indicators provided by vibration- 
based methods may be a good candidates. Using these methods, information with different level of refinement can be obtained depending on the amount of available data that is on the amount of sensors deployed on the structure and on the level of detailing of the model used for damage identification. With reference to a traditional hierarchy of damage identification [10]:

- damage detection: existence of damage

- damage localization: location of damage

- damage quantification: severity of damage

the amount of data needed to enrich the description of the damage from the first to the third level increases.

The detection of the existence of damage can be done ideally with one single sensor able to detect changes in modal frequencies. In order to to localize damage, indicators computed as functions of modal or operational shapes are needed therefore a higher number of sensors must be installed on the structure. The third level (quantification) requires also the use of a finite element.

\subsection{Step 3. Design of the SHM system}

\section{How shall the test be performed?}

Once the data needed to compute the indicators are defined, the selection of the most appropriate SHM tools and techniques to collect them can be done.

This task should be performed considering several characteristics of the SHM tools such as:

a) their feasibility for the type of asset. This may depend on several factors such as the type and size of asset (bridges, building, power plants, wind turbines, offshore structures, underground pipelines, dams, etc.), material of which the asset is made (for example some techniques may be feasible for steel but not for reinforced concrete), access restrictions related to the geometry or location of the structure, possibility to perform the test without service interruption, etc.

b) their capability to collect the data. This depends on both the type and accuracy of data required for the specific problem and on the possibility to integrate the sensing system to software tools for an efficient transmission (for example through mobile apps) and for post-processing.

c) their requirements in terms of technical capacity of the user and of the tool itself. For example, the level of the expertise of the operator, the level of technological sophistication (e.g. highpower computers), the availability of standards for the technical specifications of the instruments or of the test, the availability of easy to use (e.g. on-line) technical support for the tool, etc.

d) the cost/benefit balance. Condition monitoring may provide many benefits but can also be expensive and time-consuming therefore costs and benefits connected with the implementation of a given SHM system for a reference period should be identified and evaluated before the system is put in place [2].

\subsubsection{Design and planning of the test}

\section{Which tests, when, how?}

The final goal of SHM is to interpret the data in order to get information about the structural/operational/environmental conditions.

For this reason tests must be performed under environmental and operational conditions that are representative of the real in-service situation. Furthermore, the schedule of tests must be carefully planned in order to provide all the data needed to assess the structural condition.

The planning of the tests would be facilitated by protocols providing, for each type of test, guidance on the design of specifications of the SHM system that is on how to set the parameters of the test such as:

- The frequency of the tests

- The schedule of tests

- The transmission of data (wired/wireless)

- Structural components to test

- Considerations related to the practical implementation of the test.

In reference [7] a quite comprehensive list of all the aspects of the tests that need to be organized according to a specific protocol is suggested. The main points can be summarized as follows. The 
interested reader can refer to reference [7], freely available on the web, for a more detailed list:

- Collection of existing documentation (design and construction records, site conditions, inspection records, information stored in obsolete format), data mining (extraction of knowledge in databases);

- Planning and logistics (power and network requirements of the tests, requirement for traffic control, personnel qualification, communication and coordination plan);

- Design of the data collection grid, of structure segmentation and element identification system. This assigns an identifier to each element of the bridge according to a predefined alphanumeric system.

- List, specifications and protocols of the test to perform (material sampling, visual inspections NDTs, dynamic, monitoring), of the damage/degradation phenomena to investigate (cracks, spalls, abrasion, delamination, sulfate attack, deflection, uplift, buckling...) and of the components to test (bearings, expansion joints, deck, abutments,...)

- Definition of data type (documents, images, measures) and of storage modality (local and/or remote)

- Type of Instrumentation (sensors, testing tools, cameras) and their specifications

- Data reduction processing, analyses

- Data transmission tools (wired, wireless)

\subsubsection{Value of Information of SHM}

\section{Is it worth to perform SHM?}

Cost considerations should be carefully accounted for in the design phase of the monitoring system. The expenditure on condition monitoring should at least balance the anticipated benefits.

Cost of the monitoring system must be evaluated in terms of both tools/techniques (measurement equipment, software, frequency and type of tests) and human resources (training and labor of technicians, out-sourced work, management, administration).

The benefits can be estimated following a riskbased approach relating them to the consequences of a possible failure. Higher consequences justify higher cost of the SHM system able to provide information that may enable prompt interventions that reduce the risk of failure.

In some cases, in order to reduce costs, a stepwise approach may be implemented: low cost screening tools can be used to receive alerts about early signs of deterioration whereas more sophisticated and expensive methods are used if, following the first alert, a survey indicates the need of more accurate information to reduce the risk.

Accurate information about both the costs and benefits may be difficult to obtain due to the scarce knowledge of the consequences of failure and of their cost and to the necessity to estimate future costs over the reference monitoring period.

Furthermore, if monitoring is performed to support decisions about the need to repair or replace components, the cost of these actions should be considered in the cost/benefit analysis. If the cost of monitoring is comparable to the cost of replacement, it could be more effective to just to repair/replace the structure.

Regulatory requirements may have an important weight on the costs/benefits related to the use of a monitoring system. In some European countries the use of an SHM system allows to reduce the number of required inspections, in other countries this possibility does not exist. In identical practical conditions the benefits related to the adoption of a monitoring system may therefore result greatly enhanced if the advantages connected with its use are properly accounted for by regulations.

\subsection{Step 4. Collect data}

\section{How data should be collected? What characteristics of the data should be checked?}

Protocols must ensure that tests provide data of constant quality regardless of when, where, or by whom the data are collected [7]

To this aim both the quantity the quality of data are important and criteria to assess them should be given by the protocols.

The quality of data should be assessed according to specified confidence criteria, which can include some or all of the following aspects of data quality [9]: 
- Accuracy - Are the data reliable?

- Completeness - What is the data coverage; are there any gaps?

- Currency - Are the data sufficiently up to date?

- Consistency - Is there any contradictory data or information?

- Compatibility - Are the data produced on the same basis as other similar information?

- Credibility - Does the data align with local knowledge or typical ranges of values?

Quantity of data is also important. Collection and storing of data is costly, therefore it is important to collect only sufficient structural performance data to provide the information required by the stakeholders. The selection of the sufficient data needs also to be periodically reviewed based on management issues and changes in regulation

\subsection{Step 5. Process data and store information}

\section{How to retrieve information from data and store them?}

As mentioned before, data collected on the structure during inspections, testing or continuous monitoring are processed to compute the so-called Indicators, whose values are correlated to the structural health condition.

Permanent monitoring systems can generate huge amount of data and current technology to measure, store and transmit data does not always provide the ideal conditions for an effective processing of the measured data. Further insight and investigations are also needed to bring the methodologies for the computation of the indicators at the operational level. Many of these indicators are still at the research level and issues exist for their routinely implementation for condition monitoring [11].

Therefore the computation of indicators must always include a careful processing of data, aimed to reduce the influence of uncertainties introduced during the phases of data collection, transmission and processing. This involves several operations that can be summarized as follows [11]:

- normalization to separate the effect of environmental, operational sources from the effect of damage;
- cleansing to reject unreliable data, filtering, resampling;

- compression to reduce the dimension of measured data;

- fusion to combine information from multiple sources in an indicator so that to enhance the fidelity of the damage identification.

Once the indicator is extracted, basing on its value, a decision must be made about the (health) state of the structure in order to discriminate between the undamaged and the damaged states.

To this aim can be used statistical models derived from machine learning techniques falling into two main categories [11]:

- supervised learning algorithms when data are available from both the undamaged and damaged structure. Group classification and regression analysis are examples of such algorithms

- unsupervised learning algorithms when only data from the undamaged structure are available for training. Outlier or novelty detection methods are examples of this category.

The application of these techniques of Artificial Intelligence and other based on big data analytics allowing also the processing of data from different sources, as legacy data for example - is becoming more and more effective to treat the large amount of data made available by the new sensing technologies. Standardization can facilitate and foster a wide diffusion in the employment of such technique and technologies.

Another aspect related to the large amount of data and information provided by surveys and monitoring is the need to store them. Data or information collected by the SHM process must be first of all documented and reported, using for example collection forms. Furthermore, measures or their compact representation through indicators, should be stored in a database for future consultation. This database should collect all the possible information over the lifecycle of the structure related to different 'health' conditions (original, refurbished, after maintenance,...) that are necessary to assess structural deterioration. Protocols should be defines regarding the required 
format of data and the procedures to readily access and interrogate the database and for data unloading and uploading. These may include rules for naming the files and the folders where these are stored in order to clearly identified location and time of the collected data. As an example, in reference [7] a detailed protocol is proposed for data collected during visual inspections and NDTs.

\section{Conclusions}

In this paper are described the different steps of the design and implementation of a monitoring system for civil asset, with particular emphasis to the standardization needs. For large part of the civil structures and infrastructures the use of advanced performance monitoring systems is still at a very early stage and visual inspections are the primary tool for condition monitoring, notwithstanding the large subjectivity of the outcomes of such operations. Guidelines for the execution of the entire SHM process - from design to implementation and use of the condition monitoring system - are needed to facilitate their utilization on behalf of the different stakeholders. Standards would enable inspectors to provide more objective information about the structural condition thereby increasing the benefits connected to the use of monitoring systems.

Another aspect that should be considered is that, due to the large amount of data provided by the new sensing technologies, algorithms of artificial intelligence and big data are becoming essential to process these data.

Standardization of monitoring procedures should help to bridge the gap between theoretical research on condition monitoring and practical applications of these technique fostering their easy integration in the procedures for civil asset condition assessment.

\section{References}

[1] Del Grosso A. (2015) Activities of the Italian UNI-WG06 on Standardization in SHM. Proc of the IWSHM 2015. The 10th International Workshop on Structural Health Monitoring. DOI:10.12783/SHM2015/198.
[2] Thöns S. Limongelli M.P., Mandic Ivankovic A., Val D., Chryssanthopoulos M., Lombaert G., Döhler M., Straub D., Chatzi E., Köhler J., Wenzel H., Sørensen J. (2017). Progress of the COST Action TU1402 on the Quantification of the Value of Structural Health Monitoring. Proc of the IWSHM 2017. The 11th International Workshop on Structural Health Monitoring. September 1214, 2017, Stanford, California, USA.

[3] ISIS CANADA. 2001. Guidelines for Structural Health Monitoring, Design Manual No. 2.

[4] Mufti, AA. Guidelines for structural health monitoring. Winnipeg, MB, Canada: ISIS Canada,2001.

[5] Rucker, W, Hille, F, Rohrmann, R. Guideline for structural health monitoring. Final report, SAMCO, Berlin, 2006

[6] Yang, Y., Li, Q., \& Yan, B. (2017). Specifications and applications of the technical code for monitoring of building and bridge structures in China. Advances in Mechanical Engineering, 9(1).

[7] Hooks, J. M., \& Weidner, J. (2016). LongTerm Bridge Performance Program Protocols, Version 361 (No. FHWA-HRT-16007).

[8] U.S. Department of Transportation. Federal Highway Administration https://highways.dot.gov/bridges-andstructure/ (last consulted December 2018.

[9] Marlow D., Heart S., Stewart B., Urquarth A., Gould S., Anderson M., Cook S., Ambrose M., Madin B., Fitzgerals A. (2007) Condition assessment strategies and protocols for water and wastewater utility assets. Arwa research foundation. Water Environmental research foundation. WERF 03-CTS-20CO.

[10] Rytter, A., 1993. Vibration Based Inspection of Civil Engineering Structures. PhD Thesis. Aalborg University, Denmark.

[11] Limongelli M.P., Orcesi A. (2018) The Parameter Readiness Level for the classification of innovative performance indicators for road bridges. IALCCE Conference. Ghent, October 2018.

[12] Farrar C., Worden K. (2013). Structural Health Monitoring: a machine learning perspective. J. Wiley \& Sons Ltd. 